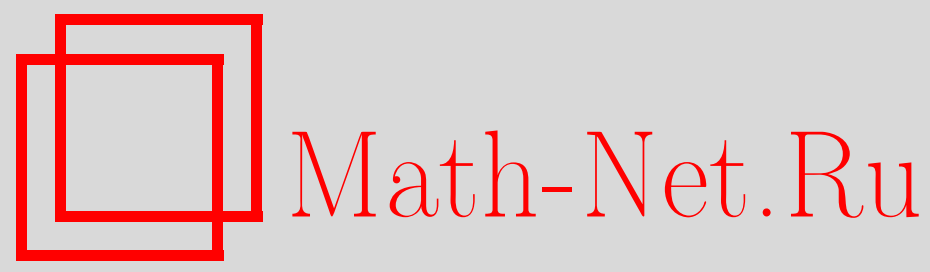

В. С. Мельник, Об оценках фрактальной и хаусдорфовой размерностей множеств, инвариантных при многозначных отображениях, Матем. заметки, 1998, том 63, выпуск $2,217-224$

DOI: https://doi.org/10.4213/mzm1268

Использование Общероссийского математического портала Math-Net.Ru подразумевает, что вы прочитали и согласны с пользовательским соглашением http://www.mathnet.ru/rus/agreement

Параметры загрузки:

IP: 3.93 .64 .190

26 апреля 2023 г., 12:38:29

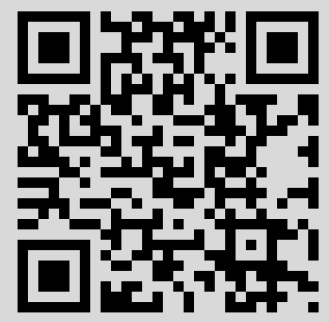




\section{ОБ ОЦЕНКАХ ФРАКТАЛЬНОЙ И ХАУСДОРФОВОЙ РАЗМЕРНОСТЕЙ МНОЖЕСТВ, ИНВАРИАНТНЫХ ПРИ МНОГОЗНАЧНЫХ ОТОБРАЖЕНИЯХ}

\section{В. С. Мельник}

В работе получены оценки хаусдорфовой и фрактальной размерностей множеств $A \subset X$, инвариантных относительно многозначных отображений $F: X \rightarrow 2^{X}$, действующих из банахова пространства $X$ на множество всех подмножеств $X$.

Библиография: 8 названий.

При изучении поведения траекторий эволюционных уравнений существенную роль играют аттракторы соответствующих однопараметрических полугрупп (или групп) нелинейных операторов. Важной характеристикой аттракторов систем уравнений в частных производных является их размерность [1]-[4]. Вычисление меры, характеризующей конечномерность динамики рассматриваемой системы, основано на оценках размерностей (топологической, хаусдорфовой, фрактальной) множеств, инвариантных относительно нелинейных преобразований гильбертовых пространств [3], [5], [6].

В данной заметке некоторые результаты О. А. Ладыженской [3], [5] по оценке размерностей обобщаются на случай многозначных отображений, что представляет интерес с точки зрения теории многозначных полудинамических систем [7].

Пусть $X$ - банахово пространство, $\mathscr{F}(X)$ - упорядоченньй по включению фильтр конечномерных подпространств $X, \operatorname{dim}_{H}(A)$ и $\operatorname{dim}_{f}(A)-$ соответственно хаусдорфова и фрактальная размерности множества $A \subset X, F: X \rightarrow 2^{X}$ - многозначное отображение $\left(2^{X}\right.$ - совокупность всех подмножеств пространства $\left.X\right), \operatorname{Dom} F=\{x \in X \mid F(x) \neq \varnothing\}$.

Напомним определения хаусдорфовой и фрактальной размерностей. Пусть $A \subset X$ и $\mathscr{U}$ - покрытие $A$ шарами $B_{r_{i}}\left(x_{i}\right)$ радиусов $r_{i} \leqslant \delta$ с центрами в точках $x_{i}$. Положим

$$
\omega_{N}(\mathscr{U}, \delta, A)=\sum_{i} r_{i}^{N}, \quad \omega_{N}(\delta, A)=\inf \omega_{N}(\mathscr{U}, \delta, A)
$$

г де инфимум берется по всевозможным покрытиям $\mathscr{U}$ множества $A$ шарами, радиусы которых не превосходят $\delta$. Хаусдорфовой мерой множества $A$ размерности $N$ назьвается число

$$
\lim _{\delta \rightarrow 0} \omega_{N}(\delta, A)=\omega_{N}(A)=\sup _{\delta \geqslant 0} \omega_{N}(\delta, A)
$$

Работа вьполненапри частичной финансовой поддержке Фонда фундаментальных исследований Украины. 
а хаусдорфовой размерностью-число

$$
\operatorname{dim}_{H}(A)=\inf \left\{N \mid \omega_{N}(A)<\infty\right\}
$$

Аналогично определяется фрактальная размерность $\operatorname{dim}_{f}(A)$, с тем лишь отличием, что покрытие $\mathscr{U}$ множества $A$ осушествляется шарами $B_{r_{i}}\left(x_{i}\right)$ при условии $r_{i}=\delta$. Очевидно, $\operatorname{dim}_{H}(A) \leqslant \operatorname{dim}_{f}(A)$.

Пусть $\Lambda=\left\{A_{i}\right\}$ - измельчение $A$, т.е. $A_{i} \subset A$ и $\bigcup_{i} A_{i}=A$, a $\{\Lambda\}=\mathscr{H}$ - некоторая совокупность таких измельчений. Положим

$$
\begin{aligned}
& \underline{\operatorname{dim}}_{f, \Lambda}(A)=\inf _{i} \operatorname{dim}_{f}\left(A_{i}\right), \quad \overline{\operatorname{dim}}_{f, \Lambda}(A)=\sup _{i} \operatorname{dim}_{f}\left(A_{i}\right), \\
& \underline{\operatorname{dim}}_{f, \mathscr{H}}(A)=\inf _{\Lambda \in \mathscr{H}} \underline{\operatorname{dim}}_{f, \Lambda}(A), \quad \overline{\operatorname{dim}}_{f, \mathscr{H}}(A)=\sup _{\Lambda \in \mathscr{H}} \overline{\operatorname{dim}}_{f, \Lambda}(A)
\end{aligned}
$$

и аналогично в случае хаусдорфовых размерностей.

Справедливы неравенства

$\underline{\operatorname{dim}}_{H, \mathscr{H}}(A) \leqslant \overline{\operatorname{dim}}_{H, \Lambda}(A) \leqslant \operatorname{dim}_{H}(A), \quad \underline{\operatorname{dim}}_{f, \mathscr{H}}(A) \leqslant \overline{\operatorname{dim}}_{f, \Lambda}(A) \leqslant \operatorname{dim}_{f}(A)$.

Для любого $A \subset X$ обозначим

$$
\|A\|_{+}=\sup _{a \in A}\|a\|_{X}
$$

ОПредЕЛЕниЕ 1. Многозначньй оператор $F: X \rightarrow 2^{X}$ называется $f$-сплющивающим на $A \subset X$, если $A \subset \operatorname{Dom} F$ и найдется селектор $f$ многозначного отображения $F$ такой, что

a) существует $l \in \mathbb{R}_{+}$такое, что

$$
\left\|F\left(y_{1}\right)-f\left(y_{2}\right)\right\|_{+} \leqslant l\left\|y_{1}-y_{2}\right\|_{X} \quad \forall y_{1}, y_{2} \in A
$$

б) найдутся $\mathscr{F} \in \mathscr{F}(X)(\operatorname{dim} \mathscr{F}=N), \delta \in(0,1 / 2)$ и непрерывный проектор П $\Pi_{\mathscr{F}}:$ $X \rightarrow \mathscr{F}$, для которых

$$
\left\|\left(1-\Pi_{\mathscr{F}}\right) F\left(y_{1}\right)-\left(1-\Pi_{\mathscr{F}}\right) f\left(y_{2}\right)\right\|_{+} \leqslant \delta\left\|y_{1}-y_{2}\right\|_{X} \quad \forall y_{1}, y_{2} \in A
$$

Для $A \subset X$ рассмотрим совокупность пар $\Theta=\left\{\left(A_{i} ; F_{i}\right)\right\}$, где $A_{i} \subset A$-ограниченное множество, $A=\bigcup_{i} A_{i}$ и $A_{i} \subseteq F_{i}\left(A_{i}\right)$, а каждое $F_{i}: X \rightarrow 2^{X}$ является $f_{i}$-сплющивающим на $A_{i}$. Такую совокупность $\Theta$ будем называть сплющивающей. Очевидно, если $\Theta_{1}$ и $\Theta_{2}$ - две сплющивающие совокупности, то $\Theta_{1} \cup \Theta_{2}$ также сплющивающая и каждая $\Theta$ (лемма Цорна) содержится в максимальной $\Theta_{\infty}$.

Пусть далее $\mathscr{H}=\{\Lambda\}$, где $\Lambda=\left\{A_{i}\right\},\left\{\left(A_{i} ; F_{i}\right)\right\}=\Theta$ - сплющивающая совокупность, $\Lambda_{\infty}=\left\{A_{i}^{\infty}\right\}$, где $\left\{\left(A_{i}^{\infty} ; F_{i}^{\infty}\right)\right\}=\Theta_{\infty}$. В этом случае получаем

$$
\underline{\operatorname{dim}}_{f, \mathscr{H}}(A)=\underline{\operatorname{dim}}_{f, \Lambda_{\infty}}(A), \quad \overline{\operatorname{dim}}_{f, \mathscr{H}}(A)=\overline{\operatorname{dim}}_{f, \Lambda_{\infty}}(A)
$$

(аналогичные равенства вьполняются и для хаусдорфовой размерности). 
Tеорема 1. Пусть $\mathscr{H}=\{\Lambda\}$, где $\Lambda=\left\{A_{i}\right\}, u\left\{\left(A_{i} ; F_{i}\right)\right\}=\Theta-$ сплющивающая совокупность для A. Тогда

$$
\overline{\operatorname{dim}}_{H, \mathscr{H}}(A) \leqslant \overline{\operatorname{dim}}_{f, \mathscr{H}}(A) \leqslant \alpha_{0},
$$

əде

$$
\begin{gathered}
\alpha_{0}=\sup _{\Lambda} \sup _{i} \alpha_{0}\left(A_{i}\right), \quad \alpha_{0}\left(A_{i}\right)=\inf _{\delta_{1 i} \in\left(0,1 / 2-\delta_{i}\right)} \Psi_{i}\left(\delta_{1 i}\right), \\
\Psi_{i}\left(\delta_{1 i}\right)=N_{i} \ln \left(\frac{\varkappa^{2} l_{i}^{2}}{\delta_{1 i}^{2}}\right) / \ln \left(\frac{1}{4\left(\delta_{1 i}+\delta_{i}\right)^{2}}\right),
\end{gathered}
$$

а $\delta_{i}$ и $l_{i}$ - константы сплющивания для $F_{i}, \varkappa-$ постоянная Гаусса.

ДокАЗАТЕЛЬСтво. Фиксируем произвольную сплющивающую совокупность $\Theta$ и рассмотрим некоторый ее элемент $\left(A_{i} ; F_{i}\right)$. Следуя [3], [5], построим последовательность покрытий $\left\{U_{k}^{i}\right\}$ множества $A_{i}$, где каждое $U_{k}^{i}$ состоит из элементов $D_{j}^{i k} \subset A_{i}$, $j=1, \ldots, n_{i}(k)$, диаметры которых $d\left(D_{j}^{i k}\right) \leqslant \varepsilon_{i k}, \varepsilon_{i k} \rightarrow 0$ при $k \rightarrow \infty$. С каждым таким покрытием связывается число $m_{\alpha_{i}}\left(U_{k}^{i}\right)=m_{i}(k) \varepsilon_{i k}^{\alpha_{i}}, \alpha_{i}>0$. Искомая последовательность покрытий $\left\{U_{k}^{i}\right\}$ конструируется индукцией по $k$. В качестве $U_{1}^{i}$ возьмем множество $A_{i}$. Пусть $U_{k}^{i}$ уже построено. Так как $A_{i}$ полуинвариантно относительно $F_{i}$, множества $\widetilde{D}_{j}^{i k}=A_{i} \cap F_{i}\left(D_{j}^{i k}\right)$ также образуют покрытие $A_{i}$. Пусть $\Pi_{\mathscr{F}}: X \rightarrow \mathscr{F}$ $\left(\operatorname{dim} \mathscr{F}=N_{i}\right.$, пространство $\mathscr{F}$ зависит, вообще говоря, от $\left.i\right)$ - непрерывный проектор, тогда любой элемент $y \in X$ может быть представлен единственным образом в виде $y=y_{1}+y_{2}$, где $y_{1} \in \mathscr{F}, y_{2} \in\left(1-\Pi_{\mathscr{F}}\right) X$. Для некоторого $v_{j}^{i k} \in D_{j}^{i k}$ рассмотрим $w_{j}^{i k}=f_{i}\left(v_{j}^{i k}\right)$, где $f_{i}-$ селектор отображения $F_{i}$, фигурирующий в определении 1 . Из оценки (2) для любого $v \in D_{j}^{i k}$ получаем

$$
\left\|F_{i}(v)-f_{i}\left(v_{j}^{i k}\right)\right\|_{+} \leqslant l_{i}\left\|v-v_{j}^{i k}\right\|_{X} \leqslant l_{i} \varepsilon_{i k}
$$

и

$$
\left\|\Pi_{\mathscr{F}} F_{i}(v)-\Pi_{\mathscr{F}} f_{i}\left(v_{j}^{i k}\right)\right\|_{+} \leqslant\left\|F_{i}(v)-f_{i}\left(v_{j}^{i k}\right)\right\|_{+} \leqslant l_{i} \varepsilon_{i k},
$$

значит $\widetilde{D}_{j}^{i k}$ лежит в шаре $B_{l_{i} \varepsilon_{i k}}\left(w_{j}^{i k}\right)$ пространства $X$ радиуса $l_{i} \varepsilon_{i k}$ с центром в точке $w_{j}^{i k}$, а проекция $\Pi_{\mathscr{F}} \widetilde{D}_{j}^{i k}-$ в шаре $B_{l_{i} \varepsilon_{i k}}^{\mathscr{F}}\left(\Pi_{\mathscr{F}} w_{j}^{i k}\right)$ пространства $\mathscr{F}$. Как известно [8],

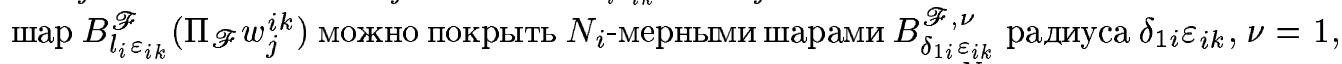
$2, \ldots, d_{i}(k, j)$, число которых $d_{i}(k, j)$ не превосходит $\left(\varkappa l_{i} / \delta_{1 i}\right)^{N_{i}}$, где $\varkappa-$ некоторая абсолютная константа (постоянная Гаусса). Тогда совокупность $\left(\Pi_{\mathscr{F}}^{-1} B_{\delta_{1 i} \varepsilon_{i k}}^{\mathscr{F}, \nu} \cap D_{j}^{i k}\right)$ образует покрытие $U_{k+1}^{i}$, являющееся измельчением $U_{k}^{i}$. Число элементов $n_{i}(k+1)$ этого покрытия не превосходит $n_{i}(k) d_{i}(k, j) \leqslant n_{i}(k)\left(\varkappa l_{i} / \delta_{1 i}\right)^{N_{i}}$. Заметим, что для любых $w_{1}, w_{2} \in \Pi_{\mathscr{F}}^{-1} B_{\delta_{1 i}}^{\mathscr{F}} \varepsilon_{i k} \cap \widetilde{D}_{j}^{i k}\left\|\Pi_{\mathscr{F}} w_{1}-\Pi_{\mathscr{F}} w_{2}\right\|_{X} \leqslant 2 \delta_{1 i} \varepsilon_{i k}$ и существуют $v_{1}, v_{2} \in D_{j}^{i k}$ такие, что $w_{1} \in F_{i}\left(v_{1}\right), w_{2} \in F_{i}\left(v_{2}\right)$ и $\left\|v_{1}-v_{2}\right\|_{X} \leqslant \varepsilon_{i k}$. Отсюда, дважды применяя оценку (3), заключаем, что $\left\|\left(1-\Pi_{\mathscr{F}}\right) w_{1}-\left(1-\Pi_{\mathscr{F}}\right) w_{2}\right\|_{X} \leqslant 2 \delta_{i} \varepsilon_{i k}$, а значит, $\left\|w_{1}-w_{2}\right\|_{X} \leqslant \delta_{2 i} \varepsilon_{i k}=\varepsilon_{i, k+1}$, где $\delta_{2 i}=2\left(\delta_{1 i}+\delta_{i}\right)$. Если $\delta_{1 i} \in\left(0,1 / 2-\delta_{i}\right)$, то $\delta_{2 i} \in(0,1)$. Тогда для числа $m_{\alpha_{i}}\left(U_{k+1}^{i}\right)$ справедлива оценка

$$
m_{\alpha_{i}}\left(U_{k+1}^{i}\right) \leqslant \eta_{i} m_{\alpha_{i}}\left(U_{k}^{i}\right),
$$


где $\eta_{i}=\left(\varkappa l_{i} / \delta_{1 i}\right)^{N_{i}}\left(2 \delta_{2 i}\right)^{\alpha_{i}}$. Выберем $\alpha_{i}$ из условия $\eta_{i}<1$, что эквивалентно неравенству

$$
0>N_{i} \ln \frac{\varkappa l_{i}}{\delta_{1 i}}-\alpha_{i} \ln \frac{1}{\delta_{2 i}}
$$

или

$$
\alpha_{i}>N_{i} \ln \left(\frac{\varkappa l_{i}}{\delta_{1 i}}\right) / \ln \left(\frac{1}{\delta_{2 i}}\right)=N_{i} \ln \left(\frac{\varkappa^{2} l_{i}^{2}}{\delta_{1 i}^{2}}\right) / \ln \left(\frac{1}{4\left(\delta_{1 i}+\delta_{i}\right)^{2}}\right) \equiv \Psi_{i}\left(\delta_{1 i}\right) .
$$

Повторяя указанную процедуру измельчения неограниченно, мы построим последовательность покрытий $\left\{U_{k}^{i}\right\}$ множества $A_{i}$ такую, что для любого $k=1,2, \ldots$ вьполняется (4) при $\eta_{i}<1$, если $\alpha_{i}>\Psi_{i}\left(\delta_{1 i}\right), \delta_{1 i} \in\left(0,1 / 2-\delta_{i}\right)$. Это означает, что $\alpha_{i}-$ мера Хаусдорфа множества $A_{i}$ - равна 0 , значит

$$
\operatorname{dim}_{f}\left(A_{i}\right) \leqslant \alpha_{0}\left(A_{i}\right)=\inf _{\delta_{1 i} \in\left(0,1 / 2-\delta_{i}\right)} \Psi_{i}\left(\delta_{1 i}\right)
$$

Поэтому утверждение теоремы 1 вытекает из определения $\overline{\operatorname{dim}}_{H, \mathscr{H}}(A)$ и $\overline{\operatorname{dim}}_{f, \mathscr{H}}(H)$. Теорема доказана.

СЛЕДСТВИЕ 1. Пусть в условиях теоремы 1 пространство $X$ гильбертово. Тогда константа $\alpha_{0}\left(A_{i}\right)$ определяется из соотношения

$$
\alpha_{0}\left(A_{i}\right)=\inf _{\delta_{1 i} \in\left(0, \sqrt{1 / 4-\delta_{i}^{2}}\right)} \Psi_{i}\left(\delta_{1 i}\right)
$$

СлЕдСтВИЕ 2. Пусть $A$ - ограниченное подмножество $X$, полуинвариантное относительно $f$-сплющивающего многозначного отображсения $F($ т.е. $A \subset F(A))$. Tогда

$$
\operatorname{dim}_{H}(A) \leqslant \operatorname{dim}_{f}(A) \leqslant \alpha_{0}
$$

$2 \partial e$

$$
\alpha_{0}=\inf _{\delta_{1} \in(0,1 / 2-\delta)} \Psi\left(\delta_{1}\right), \quad \Psi\left(\delta_{1}\right)=N \ln \left(\frac{\varkappa^{2} l^{2}}{\delta_{1}^{2}}\right) / \ln \left(\frac{1}{4\left(\delta_{1}+\delta\right)^{2}}\right) .
$$

Если при этом пространство $X$ гильбертово, то

$$
\alpha_{0}=\inf _{\delta_{1} \in\left(0, \sqrt{1 / 4-\delta^{2}}\right)} \Psi\left(\delta_{1}\right)
$$

ЗАмечАниЕ 1 . Положим $d\left(y_{1}, y_{2}\right)=\left\|y_{1}-y_{2}\right\|_{X}$. Приведенные вьше утверждения справедливы, если неравенства $(2),(3)$ заменить следующими включениями:

$$
\begin{aligned}
F\left(y_{1}\right) & \subset B^{+}\left(F\left(y_{2}\right), l d\left(y_{1}, y_{2}\right)\right) \quad \forall y_{1}, y_{2} \in A \\
\left(1-\Pi_{\mathscr{F}}\right) F\left(y_{1}\right) & \subset B^{+}\left(\left(1-\Pi_{\mathscr{F}}\right) F\left(y_{2}\right), \delta d\left(y_{1}, y_{2}\right)\right) \quad \forall y_{1}, y_{2} \in A,
\end{aligned}
$$

где

$$
\begin{gathered}
B^{+}(K, \eta)=\{y \in X \mid \operatorname{dist}(K, y) \leqslant \eta\}, \quad l \in \mathbb{R}_{+}, \quad \delta \in(0,1) \\
\operatorname{dist}(K, y)=\sup _{v \in K} d(v, y)
\end{gathered}
$$


В этом случае

$$
\alpha_{0}\left(A_{i}\right)=\inf _{\delta_{1 i} \in\left(0,\left(1-\delta_{i}\right) / 4\right)} \Psi_{i}\left(\delta_{1 i}\right), \quad \Psi_{i}\left(\delta_{1 i}\right)=N_{i} \ln \left(\frac{\varkappa^{2} l_{i}^{2}}{\delta_{1 i}^{2}}\right) / \ln \left(\frac{1}{4\left(\delta_{1 i}^{2}+\delta_{i}^{2}\right)}\right) .
$$

Если пространство $X$ гильбертово, то

$$
\alpha_{0}\left(A_{i}\right)=\inf _{\delta_{1 i} \in\left(0, \sqrt{1-\delta_{i}^{2}} / 2\right)} \Psi_{i}\left(\delta_{1 i}\right) .
$$

Рассмотрим теперь несколько иной подход к оценке хаусдорфовой и фрактальной размерностей, основанный на $N$-сжимающих многозначных отображениях. Пусть $\alpha_{1}, \ldots$, $\alpha_{k}$ - последовательность неотрицательных чисел, занумерованная в порядке убьвания, $w_{k}(\alpha)=\alpha_{1} \cdots \alpha_{k} ; B_{r}$ - шар в $X$ радиуса $r$ с центром в нуле; $\mathscr{E}(\mathscr{F} ; \alpha)$ - эллипсоид в $\mathscr{F} \in \mathscr{F}(X)(\operatorname{dim} \mathscr{F}=N)$ с центром в нуле и полуосями $\alpha_{1}, \ldots, \alpha_{N}$. В общем случае $\alpha_{i}=\alpha_{i}(y), \Pi=\Pi(y)$, т.е. каждой точке $y \in X$ соответствует свой эллипсоид и непрерьвное отображение $\Pi(y): X \rightarrow \mathscr{F}_{y}$, где $\mathscr{F} y \in \mathscr{F}(X)$ и $\operatorname{dim} \mathscr{F} y=N$. Бицилиндрами в $X$ назьваются множества вида $\mathscr{E}(\mathscr{F} ; \alpha) \oplus B_{\delta}(1-\Pi)$, состоящие из элементов $y \in X$, единственным образом представимых в виде $y=y_{1}+y_{2}$, где $y_{1} \in \mathscr{E}(\Pi ; \alpha)$, $y_{2} \in B_{\delta}(1-\Pi) \subset(1-\Pi) X$. Для $A \subset X$ положим

$$
\widehat{\alpha}_{k}=\sup _{y \in A} \alpha_{k}(y), \quad \widehat{\omega}_{k}=\sup _{y \in A} \omega_{k}(\alpha(y)) .
$$

ОПРЕДЕЛЕНИЕ 2. Пусть $A \subset X$. Многозначное отображение $F: A \rightarrow 2^{X}$ назьвается $N$-сжимающим множество $A$ в $X$, если существует $r_{0}>0$ такое, что для любого $y \in A$ найдется непрерывньй проектор $\Pi(y): X \rightarrow \mathscr{F}_{y}\left(\operatorname{dim} \mathscr{F}_{y}=N\right)$ такой, что при $r<r_{0}$

$$
F\left(\left(y+B_{r}\right) \cap A\right) \subset F(y)+r\left(\mathscr{E}(\Pi(y) ; \alpha(y)) \oplus B_{\delta(y)}(1-\Pi(y))\right),
$$

где $\alpha(y)=\left(\alpha_{1}(y), \ldots, \alpha_{N}(y)\right), \alpha_{1}(y) \geqslant \alpha_{2}(y) \geqslant \cdots \geqslant \alpha_{N}(y) \geqslant \delta(y)>0$.

Теорема 2. Пусть $A$ - компакт в гильбертовом пространстве $X$, полуинвариантный относительно $N$-сжимающего многозначного отображсения $F: A \rightarrow 2^{X}$, для любого $y_{i} \in A F\left(y_{i}\right)$ - компакт в $X, r_{0}>0$ - такое, что $F\left(y_{i}\right)$ можсно покрыть $d(i)$ шарами радиусов $r_{i} \leqslant r_{0}$, и существуют $\beta>N, k \geqslant 1, l>0$ такие, что для любого конечного набора $\left\{y_{i}\right\} \subset A$

$$
\sum_{i} r_{i}^{\beta} d(i) \leqslant k \sum_{i} r_{i}^{\beta}+l
$$

Если, кроме того,

$$
\widehat{\alpha}_{i}<\infty, \quad \widehat{\delta}=\sup _{y \in A} \delta(y)<\frac{1}{2}
$$

mo

$$
\operatorname{dim}_{H}(A) \leqslant N \ln \left(C_{N}(\varepsilon \widehat{\delta})^{-1}\left(k \widehat{w}_{N}\right)^{1 / N}\right) / \ln \left(\frac{1}{4 \widehat{\delta} \sqrt{1+\varepsilon^{2}}}\right)
$$

əде в выбирается из условия $4 \widehat{\delta} \sqrt{1+\varepsilon^{2}}<1$ и $C_{N}=1+\sqrt{N}$.

Если же $4 \sqrt{2} C_{N}\left(k \widehat{w}_{N}\right)^{1 / N}<1$, mo $\operatorname{dim}_{H}(A) \leqslant N$. 
ДокАЗАТЕЛЬСтво. Построим измельчающую последовательность покрытий $U_{k}$, $k=0,1, \ldots$, множества $A$ шарами. Пусть $U_{0}$ - набор шаров $y_{i}+B_{r_{i}}, i=1, \ldots, n\left(U_{0}\right)$, $y_{i} \in A, r_{i} \leqslant r_{0}$, объединение которых покрывает $A$. Тогда в силу полуинвариантности $A$ относительно $F$ совокупность $F\left(\left(y_{i}+B_{r_{i}}\right) \cap A\right)$ также образует некоторое покрытие множества $A$, причем

$$
\begin{aligned}
F\left(\left(y_{i}+B_{r_{i}}\right) \cap A\right) & \subset F\left(y_{i}\right)+r_{i}\left(\mathscr{E}\left(\Pi\left(y_{i}\right) ; \alpha\left(y_{i}\right)\right) \oplus B_{\delta\left(y_{1}\right)}\left(1-\Pi\left(y_{i}\right)\right)\right) \\
& \subset F\left(y_{i}\right)+r_{i}\left(P\left(\Pi\left(y_{i}\right) ; \alpha\left(y_{i}\right)\right) \oplus B_{\delta\left(y_{1}\right)}\left(1-\Pi\left(y_{i}\right)\right)\right),
\end{aligned}
$$

где $P\left(\Pi\left(y_{i}\right) ; \alpha\left(y_{i}\right)\right)$ - параллелепипед в $\mathscr{F}_{y_{i}}=\Pi\left(y_{i}\right) X\left(\operatorname{dim} \mathscr{F} y_{i}=N\right)$ со сторонами $2 \alpha_{k}\left(y_{i}\right), k=1, \ldots, N$. Покроем $P\left(\Pi\left(y_{i}\right) ; \alpha\left(y_{i}\right)\right)$ кубами $K^{i j}, j=1, \ldots, m(i)$, со стороной $2 \delta\left(y_{i}\right) \varepsilon / \sqrt{N}$. При этом диаметр $K^{i j} \oplus B_{\delta\left(y_{i}\right)}\left(1-\Pi\left(y_{i}\right)\right)$ равен $\gamma_{i}=2 \delta\left(y_{i}\right) \sqrt{1+\varepsilon^{2}}$. Множество $F\left(y_{i}\right)$ компактно, и оно содержится в

$$
\bigcup_{\nu=1}^{d(i)}\left(\xi_{i \nu}+B_{r_{i} \gamma_{i}}\right)
$$

где $d(i)$ - конечное число, а $\xi_{i \nu} \in F\left(y_{i}\right)$.

Рассмотрим множество $\left(F\left(y_{i}\right)+r_{i}\left(K^{i j} \oplus B_{\delta\left(y_{1}\right)}\right)\right) \cap A$ и предположим, что оно непусто. Тогда найдется $\nu$ такое, что

$$
A_{i j \nu}=\left(\left(\xi_{i \nu}+B_{r_{i} \gamma_{i}}\right)+r_{i}\left(K^{i j} \oplus B_{\delta\left(y_{1}\right)}\right)\right) \cap A \neq \varnothing .
$$

Множество $A_{i j \nu}$ содержится в шаре $\zeta_{i j \nu}+B_{2 r_{i} \gamma_{i}}$, где $\zeta_{i j \nu} \in A, i=1, \ldots, n\left(U_{0}\right)$, $j=1, \ldots, m(i), \nu=1, \ldots, d(i)$, и совокупность таких шаров образует новое покрытие множества $A$, которое обозначим через $U_{1}$. Покрытию $U_{0}$ соответствует число

$$
m_{\beta, r_{0}}\left(U_{0}\right)=\sum_{i=1}^{n\left(U_{0}\right)} r_{i}^{\beta}, \quad \beta>0,
$$

а покрытию $U_{1}-$

$$
m_{\beta, 2 \gamma r_{0}}\left(U_{1}\right)=2^{\beta} \sum_{i=1}^{n\left(U_{0}\right)} \gamma_{i} r_{i}^{\beta} d(i) m(i) .
$$

Нетрудно подсчитать [3], [6], что

$$
m(i)=\prod_{k=1}^{N}\left(\frac{\alpha_{k}\left(y_{i}\right) \sqrt{N}}{\delta\left(y_{i}\right) \varepsilon}+1\right) \leqslant C_{N}^{N} \frac{\omega_{N}\left(y_{i}\right)}{\left(\delta\left(y_{i}\right) \varepsilon\right)^{N}}, \quad C_{N}=1+\sqrt{N} .
$$

С учетом этого неравенства и оценки (5) при $\beta \geqslant N\left(\gamma=2 \widehat{\delta} \sqrt{1+\varepsilon^{2}}<1 / 2\right)$ получаем

$$
\begin{aligned}
m_{\beta, 2 \gamma r_{0}}\left(U_{1}\right) & =2^{\beta} \sum_{i=1}^{n\left(U_{0}\right)} \gamma_{i}^{\beta} m(i) r_{i}^{\beta} d(i) \leqslant 2^{\beta} C_{N}^{N} \sum_{i=1}^{n\left(U_{0}\right)}\left(\gamma_{i} r_{i}\right)^{\beta}\left(\frac{\omega_{N}\left(y_{i}\right)}{\delta\left(y_{i}\right) \varepsilon}\right)^{N} d(i) \\
& \leqslant 2^{\beta} C_{N}^{N} \widehat{\omega}_{N}(\varepsilon \widehat{\delta})^{-N} \sum_{i=1}^{n\left(U_{0}\right)}\left(\gamma_{i} r_{i}\right)^{\beta} d(i) \\
& \leqslant 2^{\beta} C_{N}^{N} \widehat{\omega}_{N}(\varepsilon \widehat{\delta})^{-N}\left(k \sum_{i=1}^{n\left(U_{0}\right)} \gamma_{i}^{\beta} r_{i}^{\beta}+l\right) \leqslant \eta m_{\beta, r_{0}}\left(U_{0}\right)+\sigma,
\end{aligned}
$$


где $\eta=2^{\beta} k C_{N}^{N} \widehat{\omega}_{N}(\varepsilon \widehat{\delta})^{-N}\left(2 \widehat{\delta} \sqrt{1+\varepsilon^{2}}\right)^{\beta}, \sigma=2^{\beta} l C_{N}^{N} \widehat{\omega}_{N}(\varepsilon \widehat{\delta})^{-N}$. Выберем $\beta$ из условия $\eta<1$, т.е.

$$
\beta>N \ln \left(C_{N}(\varepsilon \widehat{\delta})^{-1}\left(k \widehat{\omega}_{N}\right)^{1 / N}\right) / \ln \left(\frac{1}{4 \widehat{\delta} \sqrt{1+\varepsilon^{2}}}\right) .
$$

Таким образом, справедлива оценка (6) с $\eta<1$ и $\gamma<1 / 2$. С помощью $U_{1}$ аналогичным образом строим новое покрытие $U_{2}$, для которого

$$
m_{\beta, 4 \gamma^{2} r_{0}}\left(U_{2}\right) \leqslant \eta m_{\beta, 2 \gamma r_{0}}\left(U_{1}\right)+\sigma \leqslant \eta^{2} m_{\beta, r_{0}}\left(U_{0}\right)+\eta \sigma+\sigma .
$$

Продолжая этот процесс неограниченно, убеждаемся, что $\beta$-мера хаусдорфова множества $A$ конечна и не превосходит $\sigma /(1-\eta)$, следовательно, $\operatorname{dim}_{H}(A) \leqslant \beta$.

Предположим теперь, что $4 \sqrt{2} C_{N} \widehat{\omega}_{N}^{1 / N}<1$. Тогда $\widehat{\omega}_{N}^{1 / N}<1 /(4 \sqrt{2})$. Так как всегда $\widehat{\delta} \leqslant \widehat{\alpha}_{N} \leqslant \widehat{\omega}_{N}^{1 / N}<1 /(4 \sqrt{2})$, в формуле (5) можно положить $\varepsilon=1$, что дает $\operatorname{dim}_{H}(A) \leqslant N$.

ТЕОрема 3. Пусть выполнены условия теоремы 2 с $\widehat{\delta} \leqslant 1 /(4 \sqrt{2})$. Тогда

$$
\operatorname{dim}_{f} A \leqslant N \ln \left(C_{N} k^{1 / N} \varkappa_{N} \widehat{\delta}^{-1}\right) / \ln \left(\frac{1}{4 \sqrt{2} \widehat{\delta}}\right), \quad \varkappa_{N}=\max _{l=0, \ldots, N}\left(\widehat{\omega}_{l}^{1 / N} \widehat{\delta}^{1-l / N}\right) .
$$

Если при этом $4 \sqrt{2} C_{N} k^{1 / N} \varkappa_{N}<1$, mo $\operatorname{dim}_{f}(A) \leqslant N$.

ДокАЗАТЕЛЬСТво строится аналогично доказательству теоремы 2 с той лишш разнищей, что покрытия $U_{k}$ состоят из шаров с фиксированньпи радиусами. Так покрытие $U_{0}$ состоит из набора $y_{i}+B_{r_{0}}, i=1, \ldots, n\left(U_{0}\right)$, и ему соответствует число $m_{\beta, r_{0}}\left(U_{0}\right)=$ $r_{0}^{\beta} n\left(U_{0}\right)$. Кроме того, параллелепипед $P\left(\Pi\left(y_{i}\right) ; \alpha\left(y_{i}\right)\right)$ покрывается кубами $K^{i j}$ со стороной $2 \widehat{\delta} / \sqrt{N}$, и их число оценивается так:

$$
m(i)=\prod_{k=1}^{N}\left(\frac{\alpha_{k}(i) \sqrt{N}}{\widehat{\delta}}+1\right) \leqslant C_{N}^{N} \frac{\omega_{l(i)} \alpha\left(y_{i}\right)}{\widehat{\delta}^{l(i)}} \leqslant C_{N}^{N} \frac{\widehat{\omega}_{l(i)}}{\widehat{\delta}^{l(i)}},
$$

где индекс $l(i)$ выбирается из условий

$$
\alpha_{1}\left(y_{i}\right) \geqslant \cdots \geqslant \alpha_{l(i)}\left(y_{i}\right)>\widehat{\delta} \geqslant \alpha_{l(i)+1}\left(y_{i}\right) \quad \text { и } \quad l(i)= \begin{cases}N, & \text { если } \widehat{\delta}<\alpha_{N}\left(y_{i}\right), \\ 0, & \text { если } \widehat{\delta} \geqslant \alpha_{1}\left(y_{i}\right),\end{cases}
$$

причем $\omega_{0}=1$. При этом покрытие $U_{1}$ состоит из элементов вида $\xi_{i j \nu}+B_{2 r_{0} \gamma}$, где $\xi_{i j \nu} \in A, r_{0}$ - радиусы элементов $U_{0}, i=1, \ldots, n\left(U_{0}\right), j=1, \ldots, m(i), \nu=1, \ldots, d(i)$.

Таким образом, при $\beta \geqslant N$

$$
\begin{aligned}
m_{\beta, 2 \gamma r_{0}}\left(U_{1}\right) & =\sum_{i=1}^{n\left(U_{0}\right)}(2 \gamma)^{\beta} r_{0}^{\beta} m(i) d(i) \leqslant 2^{\beta} C_{N}^{N} \sum_{i=1}^{n\left(U_{0}\right)}\left(\gamma r_{0}\right)^{\beta} d(i) \frac{\widehat{\omega}_{l(i)}}{\widehat{\delta}^{l}(i)} \\
& \leqslant 2^{\beta} C_{N}^{N} \widehat{\delta}^{-N} \varkappa_{N}^{N} \sum_{i=1}^{n\left(U_{0}\right)}\left(\gamma r_{0}\right)^{\beta} d(i) \leqslant \eta m_{\beta, r_{0}}\left(U_{0}\right)+s,
\end{aligned}
$$

где $\eta=(4 \sqrt{2})^{\beta} k \widehat{\delta}^{\beta-N}\left(C_{N} \varkappa_{N}\right)^{N}, s=2^{\beta} l\left(C_{N} \varkappa_{N}\right)^{N} \widehat{\delta}^{-N}$.

Снова выбираем $\beta$ так, чтобы $\eta<1$, т.е.

$$
\beta>N \ln \left(C_{N} k^{1 / N} \varkappa_{N} \widehat{\delta}^{-1}\right) / \ln \left(\frac{1}{4 \sqrt{2} \widehat{\delta}}\right) .
$$

Дальнейшие рассуждения повторяют доказательство теоремы 2 . 
ЗАмЕчАниЕ 2. На основании утверждений теорем 2,3 и формулы (1) можно аналогично теореме 1 получить соответствующие оценки, используя разбиение $A=\bigcup_{i} A_{i}$, где каждому $A_{i}$ соответствует $N_{i}$-сжимаюший оператор $F_{i}: A_{i} \rightarrow 2^{X}$, относительно которого множество $A_{i}$ полуинвариантно.

\section{СПИСОК ЦИТИРОВАННОЙ ЛИТЕРАТУРЫ}

[1] Бабин А. В., Вишик М. И. Аттракторы эволюционных включений. М.: Наука, 1989.

[2] Ладыженская О.А. О нахождении минимальных глобальных аттракторов для уравнений Навье-Стокса и других уравнений с частными производными // УМН. 1987. Т. 42. №6. C. $25-60$.

[3] Ладыженская О.А. Об оценках фрактальной размерности и числа определяющих мод для инвариантных множеств динамических систем // Краевые задачи математической физики и смежные вопросы теории функций. Вып. 19. Записки научн. семин. ЛОМИ. 1987. Т. 163. C. $105-129$.

[4] Вишик М.И., Чепыжов В. В. Аттракторы неавтономных динамических систем и оценка их размерности // Матем. заметки. 1992. Т. 51. №6. С. 141-143.

[5] Л адыженская О. А. Некоторые дополнения и уточнения к моим публикациям по теории аттракторов для абстрактных полугрупп // Краевые задачи математической физики и смежные вопросы теории функций. Вып. 21. Записки научн. семин. ЛОМИ. 1990. Т. 182. С. 102-112.

[6] Douady A., Oesterlé J. Dimension de Hausdorff des attracteurs // C. R. Acad. Sci. Paris. Sér. A. 1980. V. 290. № 24. P. 1135-1138.

[7] Иваненко В. И., Мельник В. С. Вариационные неравенства и стабилизация нелинейных распределенных систем // Докл. АН УССР. Сер. А. 1989. № 2. С. 67-70.

[8] Гуревич В., Волмэн Г. Теория размерности. М., 1948.

Институт кибернетики им. В. М. Глушкова НАН Украины, г. Киев

Поступило 25.05.93

Исправленный вариант

24.04.97 